\title{
Miranda
}

Revue pluridisciplinaire du monde anglophone /

Multidisciplinary peer-reviewed journal on the English-

speaking world

$10 \mid 2014$

Images on the Move: Circulations and Transfers in film

\section{Le tambour rythmique : pour un cinéma de la régression improductive}

\section{Massimo Olivero}

URL : http://journals.openedition.org/miranda/6217

DOI : 10.4000/miranda.6217

ISSN : 2108-6559

\section{Éditeur}

Université Toulouse - Jean Jaurès

\section{Référence électronique}

Massimo Olivero, «Le tambour rythmique : pour un cinéma de la régression improductive », Miranda [En ligne], 10 | 2014, mis en ligne le 23 février 2015, consulté le 16 février 2021. URL : http:// journals.openedition.org/miranda/6217 ; DOI : https://doi.org/10.4000/miranda.6217

Ce document a été généré automatiquement le 16 février 2021.

\section{(c) (i) () $\Theta$}

Miranda is licensed under a Creative Commons Attribution-NonCommercial-NoDerivatives 4.0 International License. 


\title{
Le tambour rythmique : pour un cinéma de la régression improductive
}

\author{
Massimo Olivero
}

1 Les relations entre l'esthétique d'Eisenstein et le résultat artistique du cinéma underground ont été déjà interrogées par différents spécialistes, comme François Albera (Albera, 257), Annette Michelson ou Barthélemy Amengual. Ce dernier affirme que « les neuf dixièmes de l'underground et de l'art dit conceptuel [...] vivent » (Amengual, 588) sur le principe de la répétition, qu'Eisenstein utilise pour vérifier le saut dialectique du passage de la quantité à la qualité. En continuité avec ces recherches, nous essaierons de mettre en relation le principe esthétique de « tambour rythmique » (Eisenstein 2002, 183-193) avec trois œuvres du cinéma underground nord-américain des années soixantedix. Initialement nous analyserons les différentes conceptions du mouvement filmique dans la théorie d'Eisenstein, dont nous retracerons trois moments significatifs de sa réflexion critique, soulignant sa transformation. Dans un deuxième temps, nous croyons pertinent de formuler l'hypothèse d'une possible « circulation » de la théorie et de la pratique d'Eisenstein dans les résultats expressifs de certains films de Paul Sharits, Ernie Gehr et Michael Snow. En particulier nous chercherons à trouver des relations fécondes entre l'avant-garde eisensteinienne et l'underground surtout pour ce qui concerne la mise en crise de la représentation à travers les accélérations de vitesse du rythme filmique, la déformation plastique de l'image et les effets conséquents d'altération perceptive et psychique provoquées sur les spectateurs. Nous essaierons de retrouver non pas une application directe de la conception esthétique du cinéaste soviétique, ni des influences difficiles à justifier concrètement, mais plutôt des analogies et des possibles convergences entre Eisenstein et le cinéma expérimental américain concernant la façon de penser la nature contradictoire du mouvement filmique. 


\section{LA THÉORIE DU MOUVEMENT fILMIQUE D'EISENSTEIN : UNE PENSÉE EN DEVENIR}

2 Pour Eisenstein le mouvement cinématographique est le résultat d'un paradoxe, car il est créé à partir d'une série de photogrammes immobiles qui se superposent à la manière d'une empreinte, suivant une alternance mécanique et régulière. Si tout se passe correctement, si les photogrammes défilent à une vitesse moyenne et constante (ni trop rapide ni trop lente), ces images statiques se superposent dans l'esprit du spectateur devenant des images en mouvement. Pendant son complexe parcours théorique, il considère ce phénomène d'après trois positions différentes, voire opposées. La première fois qu'il formule cette théorie, dans La Dramaturgie de la forme filmique en 1929, il la décrit comme "deux immobilités juxtaposées [qui] donnent naissance à un concept de mobilité » et après il précise que "chaque élément successif n'est pas disposé à côté d'un autre mais par-dessus » (Eisenstein 2009, 26). Le mouvement filmique serait donc une illusion d'optique qu'Eisenstein souhaite révéler à travers une mise à nu de l'aspect mécanique du dispositif, et cela au point qu'il conduise son propre cinéma, comme le souligne Albera, «à de fréquentes mises en abyme, des dévoilements du procédé par ralentissement, accélération, répétition, 'faux-raccords' temporels ou spatiaux » (Albera, 252) comme on le voit dans la scène de la montée de l'escalier de Kérenski d'Octobre (1927). La forme filmique se charge d'exposer ce processus contradictoire de création du mouvement à partir d'objets immobiles qui s'animent, tels les lions de marbre à la fin de la séquence de l'escalier dans Le Cuirassé Potemkine, ou par le montage rapide d'une série de « cadres brefs, fixes - au photogramme » (Albera, 252). Un autre exemple typique est celui du mitrailleur dans Octobre où le montage syncopé de deux photogrammes alternés (qui montrent le militaire et la mitrailleuse), permet « l'émergence du concept de tir et non la limitation à sa représentation » (Albera, 253). Eisenstein utilise à ce propos le concept d'intervalle, c'est-à-dire la distance conflictuelle entre deux plans qu'il faut accentuer afin d'éviter la fusion des images, typique du montage canonique (l'un des thèmes centraux de sa polémique avec Koulechov et Poudovkine). Les intervalles doivent donc montrer le conflit entre les plans et faire exploser la représentation produisant le concept, l'idée au delà du visible. Eisenstein réécrit cette théorie, sous l'influence des positions esthétiques du réalisme socialiste, dans le texte Montage en 1937, renversant sa précédente position, privilégiant cette fois-ci davantage la continuité du mouvement, la fusion entre les images. Il critique en effet toute manifestation artistique qui ne donne pas l'impression de mouvement et qui dévoile le dispositif, révélant ainsi l'illusion du spectacle cinématographique. Il cite par exemple les œuvres picturales de Tintoretto, ou les dessins de Daumier, comme des cas emblématiques d'œuvres capables de « conserver l'intégrité de la perception globale de la figure [peinte] dans sa totalité » (Eisenstein 1985, 132), et cela nonobstant les diverses phases du mouvement accompli dans l'action exécutée par ces figures.

3 Le texte qui nous intéresse le plus ici est « le tambour rythmique », récemment publié dans la version russe de Méthode, son dernier livre, encore inédit en France, traitant le principe fondamental du cinéma, qu'il définit avec l'expression goethienne de Urphänomen, le phénomène à la base de sa dimension dynamique, d'un autre point de vue. Pour le dernier Eisenstein, celui des années quarante, le mouvement rythmique du dispositif cinématographique, et encore plus celui du montage, doit être employé afin 
de reconduire le spectateur aux couches primordiales de la pensée sensible, une pensée fondée sur les émotions et l'immédiateté des sensations de la conscience. Il s'agit d'après le cinéaste d'une condition que le dispositif obtient régulièrement des spectateurs, car la nature technique de l'image cinématographique, la création du mouvement à partir de l'immobilité des photogrammes, permet de produire sur le public, de façon mécanique, une prédisposition artificielle à la pensée sensible. Du reste, dit-il, "si ce n'était pas ainsi, pourquoi alors pleurerait-on face à l'écran, qui montre seulement les ombres de personnes mortes ou qui sont en train de jouer?» (Eisenstein 2002, 185). Pour lui le cinéma est, par sa structure propre, naturellement disposé à réduire le niveau de conscience intellectuelle et d'attente rationnelle du spectateur face aux images en mouvement. Dans ce texte il arrive jusqu'à affirmer que cet effet de base n'est pas le résultat d'une illusion d'optique (comme il le pensait dans les années vingt), mais plutôt le produit d'un «complexe travail psychique " (Eisenstein 2002, 186) qui renverse la traditionnelle compréhension logique et permet de percevoir l'immobile comme en mouvement. Or, grâce au "tambour rythmique ", qui frappe notre perception, se produit l'écart indispensable par rapport au plan de la conscience rationnelle, permettant ainsi de lier "le tout avec le tout", suivant donc la modalité perceptive caractéristique de la pensée primitive. Eisenstein fait ici référence à ses recherches anthropologiques et en particulier à la loi de participation des populations primitives étudiées par Lévy-Bruhl, une pensée encore étrangère aux différenciations qui seront successivement introduites par la pensée logique et analytique. Eisenstein précise ensuite que ce seul mécanisme de base du cinéma, même s'il favorise la plongée vers la pensée sensible, n'a pas le pouvoir d'éliminer totalement les strates supérieures de la conscience. Pour une complète régression psychique, permettant d'éliminer la conscience rationnelle, il faut employer un certain type de montage, qui répète sur un plan plus élevé, qualitativement supérieur, le phénomène principal. Ce montage doit se caractériser par une cadence vertigineuse, frénétique, qui exploite jusqu'au bout les possibilités intensives du tambour rythmique.

Le montage rapide deviendrait ainsi la solution expressive la plus organique par rapport au mouvement de base du cinéma, incarnant au mieux sa nature esthétique et sa référence anthropologique. Pour Eisenstein, le film, davantage que toute autre forme d'art, dispose d'une relation directe avec la pensée sensible, d'où sa force de fascination qui enchaîne et hypnotise les spectateurs. En cela le cinéma est très proche de la musique, l'art le moins tangible et le moins physique. C'est pour cette raison qu'Eisenstein est stimulé par le cinéma audiovisuel, car celui-ci dispose de deux tambours, le sonore et le plastique, qui dans leur action commune peuvent créer des synesthésies, l'un des traits les plus profonds de la pensée sensible, véritable point de convergence de sons, images, couleurs dans une sensation organique unitaire.

5 Le tambour rythmique est donc un outil formel apte à produire le phénomène de l'extase de la représentation filmique, c'est-à-dire le changement qualitatif de la forme vers un niveau expressif plus riche et plus complexe, plongeant ainsi le spectateur dans une profonde régression psychophysiologique. L'extase provoque l'indifférenciation entre les dimensions subjectives et objectives de la perception et de la compréhension humaine du réel, et rend plus aiguë la capacité de percevoir le Tout à travers les parties (pars pro toto), permettant aux couleurs de commencer

[...] à chanter et [aux] sons [ $\left.\mathrm{d}^{\prime}\right]$ avoir une forme (la synecdoque), [et oblige à réagir au] mot plein de suggestion [...] comme si le fait désigné par le mot même s'était matériellement accompli (le comportement hypnotique). (Eisenstein 2002, 187). 
Eisenstein décrit le phénomène du rythme comme lié à la dimension prélogique de la pensée, à la fois dans l'ontogenèse et la phylogenèse de l'espèce humaine : du point de vue ontogénétique il est présent dans la pensée des enfants (il évoque le "discours intérieur » de Vygotski), tout comme dans la respiration, le battement cardiaque, les mouvements de l'intestin ou les convulsions de l'orgasme. Tandis que, du point de vue de la phylogenèse historique et culturelle, il retrouve le «tambour rythmique » dans toutes les manifestations où un phénomène de possession collective ou individuelle se produit, par exemple dans les rythmes des tambours rituels des cultes vaudou ou dans les exclamations répétées frénétiquement des fanatiques de la Sainte Vierge à Lourdes, ou encore dans les cérémonies des chamanes sibériens, des derviches tourneurs, des danzantes mexicains, des gospels du sud des États-Unis, ou encore dans le rythme répétitif des exercices spirituels d'Ignace de Loyola et Thérèse d'Avila, jusqu'aux suites de Bach. Pour Eisenstein, toutes ces manifestations se caractérisent par une rythmicité fondée sur la répétition et l'itération obsessive, qui donne la possibilité d'atteindre les " couches profondes de la vie organique » (Somaini, 234) et donc la source primordiale de la créativité humaine. Malgré les différences thématiques de ces événements, le but est le même: fuir le rationnel, la perception ordinaire, éteindre la compréhension logique du réel, comme lors de l'absorption de drogues ou d'alcool (des solutions pourtant considérées par le théoricien soviétique comme trop régressives et totalement négatives dans une perspective esthétique tout comme politique).

7 Toutes ces formes fondées sur le rythme peuvent donc conduire les spectateurs à un état extatique de complète frénésie, où la volonté est paralysée et l'esprit totalement à la merci des images qui passent à toute vitesse devant leurs yeux, excitant leur perception, qui est comme subjuguée par la force de fascination du mouvement accéléré et réitéré. Le cinéma plonge ainsi artificiellement le spectateur dans cette dimension sensible, primitive, presque hypnotique, éliminant toute résistance rationnelle, comme si les strates supérieures de la conscience étaient dissoutes par l'action frappante du rythme. Cette idée de rythmicité régressive était déjà présente dans les textes des années vingt, comme dans La Quatrième dimension au cinéma (Eisenstein 1976), qui pourtant insistaient davantage sur le moment final de la synthèse intellectuelle, et voyaient seulement le montage rythmique comme un prélude à l'élaboration et à la transmission d'un message idéologique. Revenons alors à la scène citée plus haut des trois lions du Cuirassé Potemkine. Car Eisenstein, modifiant sa théorie dans Méthode, change aussi sa lecture de cet exemple. À cette étape de sa réflexion, il voit que la force idéologique de la métaphore, c'est-à-dire le peuple qui, comme le lion, se réveille et reprend conscience, est possible seulement grâce au «tambour rythmique » qui a bouleversé la perception du spectateur tout au long de la scène précédente, pendant l'avancée des soldats sur l'escalier d'Odessa. Un événement où la répétition effrayante des plans des cosaques descendant les marches trouble les yeux et les sens des spectateurs. Le rythme obsédant de la descente permet de manifester à la fois l'action et le sentiment d'égarement généré par la même action et de le faire éprouver aussi au public; c'est ainsi que se crée «ce qui est de nature à provoquer l'angoisse sur un rythme d'angoisse » (Mitry, 114). Pour le dernier Eisenstein (celui qui a vécu l'expérience mexicaine et qui écrit les pages de Méthode), le spectateur ne peut accepter l'absurdité de voir un lion de marbre qui s'anime, donc de percevoir le mouvement là où il y a quelque chose de statique, que grâce au rythme pathétique et incessant du montage qui a accompli sa régression vers l'état primordial de 
l'indistinction cognitive. Le "tambour rythmique» est alors l'outil expressif le plus adéquat pour créer une «forme d'animisme prélogique, où l'inanimé s'anime, l'immobile bouge, et nous [spectateurs] nous comportons face aux événements représentés par les images comme s'ils étaient réels » (Somaini, 234).

Il faut alors souligner que cette dernière étape atteinte par Eisenstein dans sa théorisation du rythme filmique semble vouloir libérer davantage la dimension émotionnelle et refoulée de la conscience, l'énergie pathétique et prélogique qui devait initialement servir de moteur pour produire les effets psychologiques et affectifs nécessaires à la libération politique et l'émancipation de la conscience. Or justement, dans ce texte, Eisenstein semble s'intéresser moins que d'habitude à l'aspect constructif, à la mise en forme rationnelle de l'énergie pathétique. Il nous semble en effet que, comme dans une bonne partie de Méthode, le théoricien soviétique insiste beaucoup plus sur le mouvement rythmique en tant que pur pathos, immédiateté, fusion indistincte avec l'autre, perte de soi. C'est à partir de cette conception qu'il réinterprète les images de son cinéma le plus engagé, celui des attractions intellectuelles, où il voulait surtout tenir à distance le spectateur en mettant à nu le dispositif, en privilégiant davantage le moment de la fusion des parties dans le Tout, et le moment extatique comme un retour à la condition psychique primitive. Sa réflexion sur le tambour rythmique devient donc plutôt une question esthétique, au sens propre du mot, aisthésis, c'est-à-dire visant à "rendre la perception [...] à un certain état sauvage [...] où le signifié s'étant en quelque sorte évaporé, [...] la perception, qui reconnaît [cède la place] à la sensation, qui oublie » (Noguez, 48).

\section{L'UNDERgROUND : UNE ESTHÉTIQUE DES FORMES RÉgRESSIVES}

9 Ce mouvement régressif, destructif et pulsionnel de la forme, semble donc, dans les derniers écrits d'Eisenstein, ne plus arriver à atteindre sa dimension productive, mais plutôt, dans ses moments de plus forte intensité, plonger les spectateurs dans l'indistinct et dans des conditions proches de l'ivresse, de la transe, de l'hystérie, sans reconversions ultérieures ou sublimations d'ordre idéologique. Une dimension excessive de la forme filmique donc, qui peut se retrouver aussi ailleurs, au-delà de l'expérience eisensteinienne, et qui permet de rapprocher sa dernière conception du «tambour rythmique» d'autres œuvres qui emploient le montage rapide ou, plus généralement, un rythme frénétique des images, avec pour conséquence une mise en crise de toute compréhension intellectuelle. Nous pensons à certains films de l'underground nord-américain (ou cinéma expérimental américain) qui développent et poursuivent jusqu'à l'excès les intuitions du «tambour rythmique ", car leur esthétique, elle aussi, provoque l'étourdissement perceptif grâce à la création d'images déchaînées, sans stabilité et sans limitations d'ordre mimétique. Le rapprochement entre Eisenstein et l'underground, malgré de différences évidentes, n'est pas du tout injustifié sur le plan esthétique car, comme soulignait déjà dans les années soixante-dix Annette Michelson : «Eisenstein, dans un magnifique bond de l'imagination, a exprimé sur papier la teneur essentielle, la forme, l'élan et la stratégie du Cinéma Indépendant américain des deux dernières décennies" (Michelson, 41). Eisenstein pouvait seulement laisser entrevoir les possibilités expressives d'une esthétique en même temps organique et pathétique, car celle-ci était conditionnée par des raisons d'ordre 
culturel et politique (autre que la censure). Or, le cinéma expérimental pouvait les amener à accomplissement, en "radicalisant certaines expériences " (Albera, 257) et plusieurs de ses principes formels, comme celui qui nous intéresse du «tambour rythmique ». En effet, ces cinéastes expérimentaux arrivent à faire exploser la structure représentative et figurative de l'œuvre filmique, structure qu'Eisenstein avait seulement voulu déformer, élargir jusqu'au maximum de ses possibilités, mais non pas dépasser totalement. Le cinéma expérimental donc, d'une certaine façon, trahit la lettre de la théorie eisensteinienne, mais en réalise l'esprit, car il la conduit, pour utiliser le langage du même théoricien, à une étape qualitativement supérieure, à un niveau d'intensité extatique que ce dernier n'aurait pas pu atteindre. Donc, même si l'on peut penser qu'Eisenstein n'aurait pas accepté ces résultats, car il les aurait sûrement considérés comme inorganiques, trop détachés de toute relation au sens, au réel et au temps, l'underground permet de révéler, de mettre au jour la véritable essence de la théorie de l'extase car il élargit, développe et exploite les formes filmiques en suivant la même direction, c'est-à-dire "la sortie de la représentation". Notre intérêt est alors de retrouver ce chemin, cette méthode de l'extase de la représentation, à partir $\mathrm{du}$ principe formel $\mathrm{du}$ «tambour rythmique» qui, nous l'avons vu précédemment, ne se limite pas au seul effet de montage, mais concerne aussi toute manifestation rythmique fondée sur la répétition obsédée. Une méthode qui s'est pleinement développée selon nous dans (au moins) trois cas symptomatiques, qui proviennent tous du courant du film dit «structurel » par P. Adams Sitney.

Le premier exemple est Serene Velocity d'Ernie Gehr (1970), un film qui travaille la force rythmique du zoom. Il a été parfaitement décrit par Sitney :

Gehr installa une caméra dans le couloir d'un immeuble banal et prit des photos, image par image, changeant de pose à chaque fois et les groupant par séquence de quatre images. Entre chacune des séquences, il change la position du zoom. Il le fait passer, au cours du film, de deux positions moyennes, voisines l'une de l'autre, aux extrémités de l'éloignement et du rapprochement. [...] Le film apparaît aux yeux d'un spectateur comme la description d'un crescendo rythmique, car, bien que la position de la caméra reste la même, tout comme son champ visuel, le mouvementillusion du zoom, depuis ce qui se révèle être une pulsion vibratoire jusqu'à un énergique mouvement de va-et-vient, crée une tension optique croissante qu'accompagne la destruction progressive de l'illusion spatiale par l'accent mis sur les angles droits du couloir devenus une grille à deux dimensions à la suite des variations de zoom (Sitney, 31).

11 Ce qui trouble le regard et notre perception, dans les moments les plus frénétiques du rythme, est la mise sous tension de la surface plate et neutre de l'image, qui semble sur le point d'éclater, de sortir de l'écran, d'imploser, ou de se replier sur elle-même. Cet effet, obtenu par un tambour rythmique purement mécanique, à travers une cadence saccadé des photogrammes, réalise, sur un plan plus complexe et plus abstrait, les mêmes résultats cherchés par Eisenstein lorsqu'il essayait, à travers la composition du cadre, à produire un effet à la fois centripète et centrifuge sur le regard des spectateurs. Or, si les plans réalisés par Eisenstein, par exemple dans Que viva Mexico! (1932), grâce au 28mm, voulaient en même temps nous plonger à l'intérieur de l'image et nous en expulser, Serene Velocity nous donne grâce au rythme syncopé la même sensation de déséquilibre, d'oscillation frénétique d'un espace à l'autre, d'attraction et de répulsion d'un lieu indéterminé. Nous avons ici un exemple d'images fixes et insignifiantes qui arrivent à produire un état hypnotique, une sollicitation émotive exceptionnelle grâce au clignotement effectué par le montage. Ce qui est accentué dans 
le cinéma expérimental par rapport à l'extase eisensteinienne est la dimension irrationnelle, l'immédiateté d'une émotion qui est produite par les seuls effets plastiques et graphiques du plan et par la violence empathique du rythme, sans autre élément intermédiaire. Car chez Eisenstein les formes expressives aptes à produire l'extase, tel le tambour rythmique ou la composition déformée du cadre, devaient s'unir, de façon organique, aux éléments représentatifs présents dans la mise en scène, comme les visages des Mexicains mis en relation avec les pyramides et les statues de leurs ancêtres Mayas. Une dimension organique que le cinéma underground abandonne en faveur d'une intensification $d u$ caractère pathétique et pulsionnel de l'image. Comme Raymond Bellour le souligne dans son livre Le corps du cinéma, l'émotion produite par les films expérimentaux semble être "plus pure, au sens où elle est rapportée presque sans médiation aux matières comme aux principes structuraux qui l'organisent » (Bellour, 329).

12 Les principes de répétition et de frénésie au cœur de l'action du tambour rythmique se retrouvent aussi dans un film de Michael Snow, Back and Forth (1969). Toutefois, dans ce film, au lieu d'employer le montage, le cinéaste québécois génère le même effet optique du tambour rythmique grâce au balancement scandé par le mouvement d'oscillation contradictoire de la caméra, qui se dirige dans toutes les directions et arrive à une vitesse considérable, plongeant la représentation dans l'abstraction et la perception du spectateur dans l'indéterminé. Nous sommes totalement emportés par le rythme obsédant de la caméra au point de risquer de perdre tout repère spatio-temporel, et donc toute conscience d'une distance, d'une séparation entre corps et image. Nous citons à ce propos Sitney, qui affirme que

à mesure que le film progresse, le mouvement prend de la vitesse, de sorte que la pièce donne désormais l'illusion d'un espace purement optique, comme celui de Serene Velocity, et non plus d'un espace physique et tactile, lieu possible d'une activité humaine. [...] Dans la coda du film, le mouvement successif de va-et-vient de la bande elle-même devient thématique, car le film se déroule en surimpression dans deux directions à la fois, définissant ainsi un espace-temps uniquement cinématographique (Sitney, 33).

Le film produit sur le spectateur l'expérience à la fois de l'excitation et de la narcose, la fascination de perdre le contact avec le monde et la nécessité de se détacher de ce rythme infernal. Le cadre semble toujours sur le point d'éclater, par l'action simultanée d'une régression centripète vers la matière informe - qui correspond à la perte de soi - et d'une expulsion centrifuge hors des limites de l'image, qui nous fait retrouver une distance par rapport au film. Les spectateurs sont, comme Sitney l'affirme, dans un nouvel espace-temps, qu'on pourrait comparer à une version plus extrême de la condition extatique théorisée par Eisenstein, où se vérifie d'ailleurs, mais avec une moindre intensité, le même mouvement contradictoire. Back and Forth semble prendre à la lettre ce principe esthétique et psychologique, étant donné que grâce à cette expérience filmique les spectateurs vont au-delà de toute représentation, même si c'est seulement en "revenant" à l'intérieur de la représentation qu'il est possible de comprendre rationnellement cette sensation de sortie. Le film de Snow demeure à la fois abstrait et figuratif et n'arrive jamais à abandonner la représentation, même si sa structure et son rythme essaient de s'éloigner d'elle et de l'abandonner définitivement. Cela veut dire que le cinéma de l'extase, même le plus radical comme celui de l'underground, pour montrer la sortie de la représentation, est toujours obligé de représenter cette sortie. Une telle condition psychique paradoxale est bien illustrée par 
la double flèche inspirant le titre du film, qui ressemble beaucoup au dessin de Saul Steinberg, qu'Eisenstein utilise dans La Non-Indifférente Nature (Eisenstein 1976, 406) pour visualiser la condition contradictoire de l'espace-temps dans une représentation en état extatique. Il s'agit d'une image montrant un panneau de signalisation sur lequel nous lisons l'inscription "exit", mais qui indique en même temps deux directions opposées. Rappelons que cette figure paradoxale apparaît aussi dans un autre film expérimental, L'homme à la caméra de Dziga Vertov (1929), encore une fois comme si elle était la conséquence d'un rythme déchaîné du montage, à la fin d'une série de fragments montés à une vitesse frénétique, comme s'il s'agissait de la figure cristallisant le principe rythmique qui est au cœur du mouvement cinématographique, dans ce conflit toujours présent entre immobilité et dynamisme.

Le troisième cinéaste underground que nous prenons en considération est Paul Sharits, qui travaille lui aussi sur la pulsation rythmique de l'image. De plus, il s'agit du seul des cinéastes ici convoqués qui cite directement les travaux théoriques d'Eisenstein, notamment le premier texte où apparaît la notion de "tambour rythmique ", c'est-àdire La Quatrième dimension au cinéma (Sharits, 5). Comme dernier exemple, nous prendrons un film qui résume la plupart des thèmes traités ici. Il s'agit d'Epileptic Seizure Comparison (1976), film qui aborde l'épilepsie en tant que sujet, mais aussi à travers sa construction formelle, notamment par l'emploi du flicker, un battement visuel, un assombrissement cadencé qui intervient sur l'image en déchirant son propre mouvement. L'œuvre est structurée en trois parties: dans la première, une série de plans est envahie par des couleurs pures qui clignotent grâce à l'utilisation du flicker. Cette partie voudrait plonger le spectateur dans la « dimension plastique et affective de la couleur» (Beauvais, 9), une couleur indépendante, qui se détache de toutes les surfaces des objets, mais qui en même temps exprime formellement un aspect du thème de la crise épileptique. La première phase «travaille selon des rythmes et des enchaînements de couleurs qui font de l'expérience du film une épreuve visuelle autant qu'une prise de conscience quant à la spécificité de l'expérience proposée » (Beauvais, 9). Sharits a utilisé dans beaucoup de ses œuvres la couleur détachée de tout rapport figuratif et descriptif, mais ici se configure une ambiguité évidente: d'un côté la couleur est utilisée d'une façon totalement inorganique, séparée de toute référence et sans objets ou surfaces qui la circonscrivent; mais d'un autre coté, c'est une couleur pleinement "mise en image", c'est-à-dire en relation organique avec le thème central de l'œuvre. Cette première phase exprime une crise, donnant la sensation de s'enfoncer vers la dimension la plus régressive de la conscience ou de la psyché, afin de créer une nouvelle image-limite capable de modeler la condition informelle du chaos qu'Eisenstein considérait comme une partie essentielle du moment prélogique. Sharits réalise donc deux des objectifs théorisés par Eisenstein et apparemment inconciliables : réussir à sentir visuellement le thème central de l'œuvre et en même temps atteindre les pulsions inconscientes et profondes, ingouvernables, qui appartiennent à la matière de la couleur.

Dans la deuxième phase, aux distorsions sonores et aux pulsations violentes des flickers, s'ajoutent les contorsions des patients, qui rendent le film inquiétant et parfois insoutenable. Comme s'ils étaient des Laocoons modernes, les patients sont mis sous tension à travers des fils électriques qui agitent et déforment leurs corps par des décharges d'électrochocs. Les mouvements deviennent convulsifs, les visages se déforment en d'horribles grimaces, les yeux sont révulsés, les mains s'agitent dans le 
vide. Nous retrouvons encore en action cette simultanéité contradictoire qui fait coexister mobilité et paralysie dans l'action répétée du tambour rythmique. Cette contradiction est encore plus accentuée sur le plan formel par l'utilisation du flicker qui, avec ses pulsations intermittentes produit une « tension entre le temps qui s'écoule et le temps arrêté " (Krauss, 91). Nous avons cité la statue du Laocoon (la copie romaine en marbre d'après un original grec, réalisé vers 50 après J.-C., qui se trouve à Rome aux musées du Vatican) car elle incarne au plus haut niveau le principe ambivalent du mouvement qui naît de l'immobilité, s'agissant comme dit Goethe d'une sculpture en mouvement qui est en train de passer "d'un état à un autre". Une tension que chacun peut comprendre au mieux en clignant des paupières :

Afin de bien saisir le dessein du Laocoon, le mieux est de se placer face à lui, à une distance convenable, les yeux fermés. Qu'on les ouvre ensuite pour les refermer immédiatement après, et on verra le marbre tout entier en mouvement; on craindra de trouver changé le groupe entier en les rouvrant (Goethe 1996, 169-170).

Le film de Sharits montre aussi deux mouvements contradictoires qui s'affrontent en un seul corps, celui du film même, où la dynamique du mouvement et la stase s'opposent. Sharits affirme à ce propos que :

[...] ma préoccupation majeure concerne la dynamique d'une image humaine. Par exemple, dans Epileptic, ce qui ressort, ce sont les mouvements et la dynamique des convulsions qui agitent les figures. La couleur et le rythme du film, la clarté et l'obscurité dans les pulsations de l'image, les aspects formels, servent à souligner les contorsions des figures (Lebensztejn, 81).

Le « tambour rythmique » traverse ici toutes les sections de la construction formelle, il se retrouve dans les figures des patients soumis au déchaînement de la crise épileptique tout comme dans les distorsions visuelles et temporelles produites par le flicker (sans oublier la violence déterminée par le bruit sonore). Le film aboutit à une mise en crise de tous ses éléments, dont les formes sont déstabilisées, tordues, rendues pathétiques. Cet effet se multiplie dans la dernière partie qui fusionne ensemble les phases précédentes, produisant un saut qualitatif supplémentaire. Ce moment d'extrême tension bipolaire s'obtient non pas par la somme des deux phases précédentes, mais par leur produit, qui augmente l'impact émotionnel du film. Les corps des malades de la phase $B$ entrent en rapport avec les couleurs pures de la phase $A$ et génèrent un mélange inextricable d'images figuratives et d'images abstraites. Un véritable bond qualitatif se produit, un passage de la figuration à la non figuration, du coloré à l'incolore, du mouvement à l'arrêt sur image, dans une tension contradictoire qui arrive à décliner en une nouvelle forme la "simultanéité contradictoire" (DidiHuberman, 297) propre à toute image de l'extase.

19 Le « tambour rythmique ", conçu initialement, dans les écrits des années vingt, comme une solution expressive apte à libérer les consciences afin de mieux les disposer à une nouvelle conversion idéologique, provoquerait plutôt une exaspération des facultés de représentation filmique et des facultés cognitives et logiques du spectateur. Eisenstein semble conscient de cela dans son texte de Méthode, mais n'arrive pas dans sa pratique à mettre en acte tout son potentiel. Il est du reste encore davantage lié à une forme d'art construite sur le principe de la médiation dialectique des parties, qui recherche le sens à travers la sensation, le logos par le pathos. Ce sont les cinéastes underground qui ont pu fonder davantage leur esthétique sur le principe de l'immédiateté, qui permet d'exhiber la force destructive du sensible, sans se laisser réduire par le discours, le texte et la signification. Le rythme frénétique des images analysées semble donc mettre 
en évidence les limites de la forme représentative ainsi que de la perception humaine. Il s'agit d'une solution expressive fondée sur l'altération et la dissemblance, qui conduit là où la pratique esthétique de l'œuvre filmique amène un processus psychique de régression du spectateur. Le "tambour rythmique » altère et déréalise le réel, fatigue nos yeux et nous plonge dans un flux informe qui fait chanceler notre raison, nos sens et émotions.

\section{BIBLIOGRAPHIE}

Albera, François. Eisenstein et le constructivisme russe, Stuttgart dramaturgie de la forme. Lausanne : L’Âge d'homme, 1990.

Amengual, Barthélemy. Que viva Eisenstein! Lausanne : L’Âge d'homme, 1980.

Aumont, Jacques. Montage Eisenstein. Paris : Éditions Images modernes, 2005.

Beauvais, Yann. « Figmen. » In Paul Sharits. Ed. Yann Beauvais. Dijon : Les presses du réel, 2008. XX-XX.

Bellour, Raymond. Le corps au cinéma, hypnoses, émotions, animalités. Paris : POL, 2009.

Brenez, Nicole. Cinémas d'avant-garde. Paris : Cahiers du cinéma, 2006.

Didi-Huberman, Georges. L'image survivante : histoire de l'art et temps des fantômes selon Aby Warburg. Paris : Éd. de Minuit, 2002.

Eisenstein, Sergueï M. La Non-indifférente nature 1. Paris : Union générale d'éditions 10/18, 1976.

---. « La Quatrième dimension au cinéma »In Le film : sa forme/son sens. Paris : Christian Bourgois, 1976.

---. Teoria generale del Montaggio. Venise : Marsilio, 1985.

---. « Le Tambour rythmique. » In Metod 1. Moscou : Muzej Kino, 2002.

---. « Dramaturgie de la forme filmique. » In Cinématisme : peinture et cinéma. Ed. François Albera et al. Dijon : les Presses du réel, Paris : Kargo, 2009.

Goethe, Johann W. « Sur Laocoon ». Écrits sur l'art. 1798. Paris : Flammarion, 1996.

Krauss, Rosalind. « Paul Sharits. » Trafic : 29 (1999) : 84-95.

Lebensztejn, Jean-Claude. “Interview with Paul Sharits." In Paul Sharits. Ed. Yann Beauvais. Dijon :

Les presses du réel, 2008.

Michelson, Annette. «Scène de l'action espace du mouvement. La crise de la représentation cinématographique. » In Une histoire du cinéma. Ed. Peter Kubelka. Paris : Centre national d'art et de culture Georges Pompidou, 1976. 38-44.

Mitry, Jean. S. M. Eisenstein. Paris : J.-P. Delarge, 1978.

Noguez, Dominique. «Qu'est-ce que le cinéma expérimental ? Sa situation en France. » In Une histoire du cinéma. Ed. Peter Kubelka. Paris : Centre national d'art et de culture Georges Pompidou, 1976. 45-52. 
Noguez, Dominique. Une renaissance du cinéma : le cinéma underground américain : histoire, économie, esthétique. Paris : Éditions Paris Expérimental, 2002.

Sharits, Paul. « Entendre : Voir. » 1975. In Paul Sharits. Paris : Éditions Paris Expérimental, 2002. 3-8.

Sitney, P. Adams. « Tableau historique. » In Une histoire du cinéma, Ed. Peter Kubelka. Paris : Centre national d'art et de culture Georges Pompidou, 1976. 9-37.

---. Le film structurel. Paris : Éditions Paris Expérimental, 2006.

Somaini, Antonio. Ejzenštejn, il cinema, le arti, il montaggio. Turin : Einaudi, 2011.

Vygotski, Lev. Pensée et langage. 1925. Paris : Messidor, Éditions sociales, 1985. d

\section{RÉSUMÉS}

Le texte interroge la théorie sur le mouvement cinématographique de Sergueï Eisenstein et les possibles relations de son esthétique avec le cinéma underground nord-américain des années 1970. En particulier il s'agira de proposer le principe formel du «tambour rythmique », conçu par le cinéaste soviétique pour analyser le montage de ses œuvres d'avant-garde, comme clé de lecture de l'écriture expérimentale de Back and Forth de Michael Snow, Serene Velocity d'Ernie Gehr et Epileptic Seizure Comparison de Paul Sharits.

Drawing upon Eisenstein's theory of cinematographic movement this article relates the aesthetic principle of "rhythmic drum" that was initially devised to analyse the editing techniques of his own avant-garde productions, to three North American underground films from the 1970s, Back and Forth by Michael Snow, Serene Velocity by Ernie Gehr and Epileptic Seizure Comparison by Paul Sharits.

\section{INDEX}

Keywords : underground, rythme, régression, mouvement

Mots-clés : rythme, régression, mouvement

\section{AUTEURS}

\section{MASSIMO OLIVERO}

Paris 3 Sorbonne-Nouvelle

Doctorant

olivero.massimo@yahoo.fr 